\title{
Improved rotation-activity-age relations in Sun-like stars
}

\author{
Jorge Meléndez, Leonardo A. dos Santos and Fabrício C. Freitas \\ Universidade de São Paulo, IAG, Departamento de Astronomia \\ Rua do Matão 1226, 05508-090 São Paulo, SP, Brazil \\ email: jorge.melendez@iag.usp.br
}

\begin{abstract}
The evolution of rotational velocity and magnetic activity with age follows approximately a $t^{-1 / 2}$ relation, the famous Skumanich law. Using a large sample of about 80 solar twins with precise ages, we show departures from this law. We found a steep drop in rotational velocity and activity in the first 2-3 Gyr and afterwards there seems to be a shallow decrease. Our inferred rotational periods suggest that the Sun will continue to slow down, validating thus the use of gyrochronology beyond solar age. The Sun displays normal rotational velocity and activity when compared to solar twins of solar age. We also show that stars with exceedingly high stellar activity for their age are spectroscopic binaries that also exhibit enhanced rotational velocities and chemical signatures of mass transfer.
\end{abstract}

Keywords. Sun: activity, Sun: rotation, stars: activity, stars: rotation, techniques: spectroscopic

\section{Introduction}

Solar twins are defined as stars with effective temperatures within $100 \mathrm{~K}$, and $\log g$ and $[\mathrm{Fe} / \mathrm{H}]$ within 0.1 dex around the Sun's values (Ramírez et al. 2014). The first solar twin, 18 Sco, was discovered in 1997 by Porto de Mello \& da Silva (1997, see also Soubiran \& Triaud 2004). Only in the mid-2000s new solar twins were identified and in the last decade their number has grown to about a hundred (Mahdi et al. 2016; Yana Galarza et al. 2016b; Porto de Mello et al. 2014; Datson et al. 2014, 2012; Ramírez et al. 2014; Meléndez et al. 2014b, 2009, 2006; do Nascimento et al. 2013; Takeda \& Tajitsu 2009; Pasquini et al. 2008; Takeda et al. 2007; Meléndez \& Ramírez 2007; King et al. 2005).

An important application of solar twins is to set the zero-point of fundamental calibrations in astrophysics (Datson et al. 2015, 2012; Casagrande et al. 2014, 2010; Ramírez et al. 2012; Meléndez et al. 2010), but many other studies have been possible thanks to the precise stellar parameters $\left(\sigma\left(\mathrm{T}_{\text {eff }}\right) \leqslant 10 \mathrm{~K}, \sigma(\log g) \leqslant 0.02\right.$ dex $)$ and chemical abundances $(\sigma \sim 0.01$ dex) that can be achieved in these stars (e.g., Nissen 2015; Bedell et al. 2014; Monroe et al. 2013; Kiselman et al. 2011). One landmark result of the first high precision studies showed that the Sun has a distinct abundance pattern (Meléndez et al. 2009; Ramírez et al. 2009, 2010). Strikingly, this peculiar pattern may be related to the formation of rocky planets in our solar system (Chambers 2010). In a related investigation, Tucci Maia et al. (2014) showed that the binary system of solar twins 16 Cyg displays chemical abundance differences likely due to planets, and other planet-hosting wide binary systems composed of solar-type stars also show distinct abundances probably due to planets (Teske et al. 2016a,b; Adibekyan et al. 2016; Saffe et al. 2016; Ramírez et al. 2015; Biazzo et al. 2015).

Solar twins have also been crucial to obtain reliable isochrone ages (Tucci Maia et al. 2016; Nissen 2015), determine improved mass and radius of benchmark planets (Bedell et al. 2016), study the possible effects of radioactive elements on rocky planet interior 


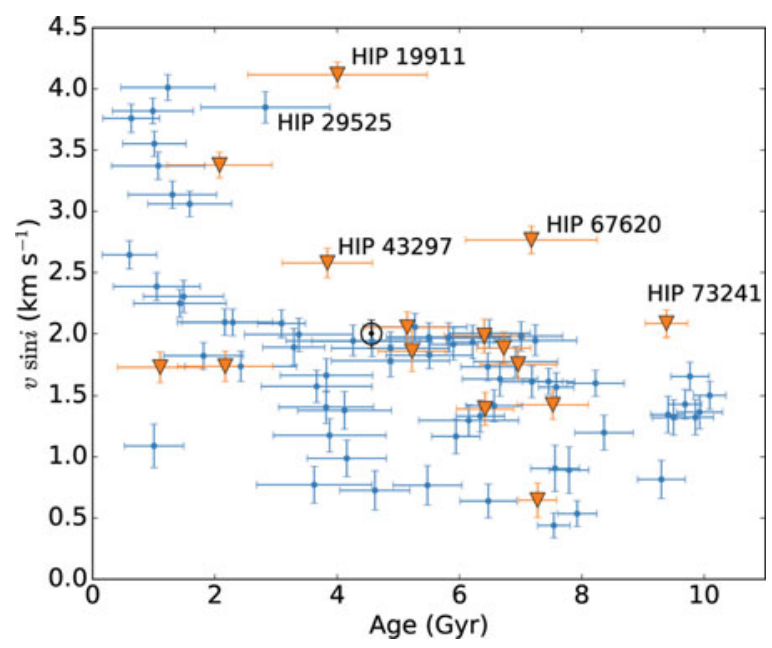

Figure 1. Projected rotational velocities in solar twins vs. age. The Sun is shown with its usual symbol and the solar twins by blue points, except the spectroscopic binaries that are shown by inverted orange triangles (outliers are labeled). Figure from dos Santos et al. (2016).

dynamics and habitability (Unterborn et al. 2015), infer the effects of mass transfer by the progenitors of white dwarfs (Desidera et al. 2016; Schirbel et al. 2015), observe stellar oscillations to constrain their masses and ages (Metcalfe et al. 2012; Li et al. 2012; Bazot et al. 2011), inspire new stellar models including episodic accretion (Baraffe \& Chabrier 2010), observe depletion of the light elements Li (Carlos et al. 2016; Monroe et al. 2013; Baumann et al. 2010) and Be (Tucci Maia et al. 2015) and model their decrease through non-standard stellar models (Andrássy \& Spruit 2015; Castro et al. 2011; Denissenkov 2010; do Nascimento et al. 2009), develop chemical clocks through the [Y/Mg] and [Y/Al] ratios (Tucci Maia et al. 2016; Spina et al. 2016; Nissen 2016, 2015), and to uncover the nucleosynthetic history of our Milky Way empirically through cosmic phylogeny (Jofré et al. 2017) or through the detailed study of abundance ratios (e.g., Nissen 2016, 2015; Spina et al. 2016; Yana Galarza et al. 2016a; Meléndez et al. 2014a).

Since 2011 we have been monitoring about 70 solar twins for planets at the ESO La Silla Observatory (Meléndez et al. 2015; Ramírez et al. 2014), and have found a Jupiter-twin (Bedell et al. 2015) and a super-Neptune and super-Earth (Meléndez et al. 2017). Using the individual and combined HARPS spectra obtained for our sample, and additional spectra of 9 solar twins gathered by other ESO programs, we studied the evolution of rotational velocity and magnetic activity using about 80 solar twins with precise stellar ages measured by a differential comparison of our precise stellar parameters with isochrones (Tucci Maia et al. 2016). We also assess how common is the Sun by a comparison with solar twins.

\section{The Skumanich law and the Sun among stars}

More than four decades ago, Skumanich (1972) showed that there is a decline of rotational velocity $(v)$ and activity with age $(t)$, following approximately a $t^{-1 / 2}$ relation, the famous "Skumanich law". Impressively, this widely used relation was obtained using only a few data points.

Under some assumptions, the $v \propto t^{-1 / 2}$ relation can be derived from the angular momentum loss due to stellar winds (e.g., Soderblom 1983; Kawaler 1988). However, there are evidences for departures from the Skumanich law. The work by Pace \& Pasquini 


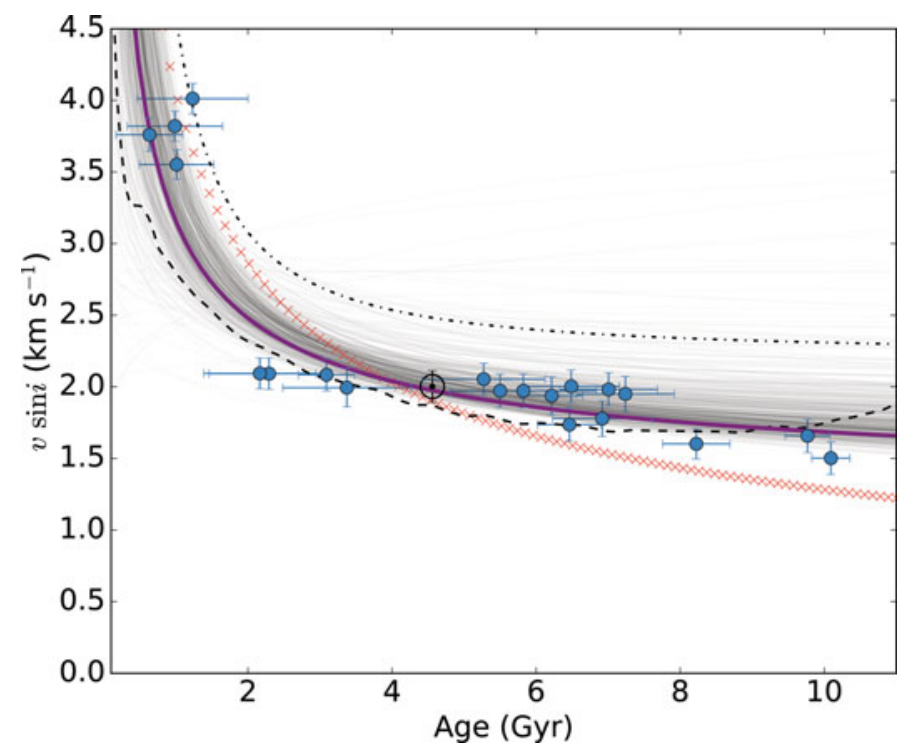

Figure 2. Projected rotational velocities in solar twins of the selected sample vs. age. The Sun is shown with its usual symbol. Our best fit (solid line) and the Skumanich law (red symbols) are shown. Figure from dos Santos et al. (2016).

(2004) suggests a steep decline of rotational velocities and stellar activity in the first 1-2 Gyr and then a flat trend with age. Our large sample of solar twins is ideal to verify whether the decay of rotational velocity and activity is smooth, or if they behave as suggested by Pace \& Pasquini (2004).

Many previous works sought to evaluate the place of the Sun among other stars (e.g., Gustafsson 2008, 1998; Shapiro et al. 2013). This is of fundamental importance because the Sun is used to validate stellar models, so we must know whether the Sun is a regular star or an oddball. Unfortunately, conclusions by previous studies could be hampered by biased samples, and the Sun has not been compared to strictly Sun-like stars (e.g., Robles et al. 2008). And, even when the Sun is compared to similar stars, there is the issue of systematics due to the observations of the Sun and the stars being carried out with different instruments. This is clearly shown in a recent study by Egeland et al. (2016), where the Sun's magnetic activity $S$-index is shown to be lower than previously estimated.

In our work the Sun and the comparison stellar sample are analysed using spectra obtained by the HARPS spectrograph at the ESO La Silla Observatory, all gathered with the same high resolution $(R=115000)$ and at high signal-to-noise ratio $(S / N)$ for our planet search around solar twins (Meléndez et al. 2015). Furthermore, our reference sample of solar twins have approximately solar mass and metallicity, therefore they follow evolutionary paths similar to the Sun. Thus, our sample is ideal to verify if the Sun is normal in its activity level and rotational velocity.

\section{Rotational velocities}

In order to determine the projected rotational velocities in our sample, we fit the line profiles considering instrumental, rotational and macroturbulent broadening, as described in dos Santos et al. (2016). 


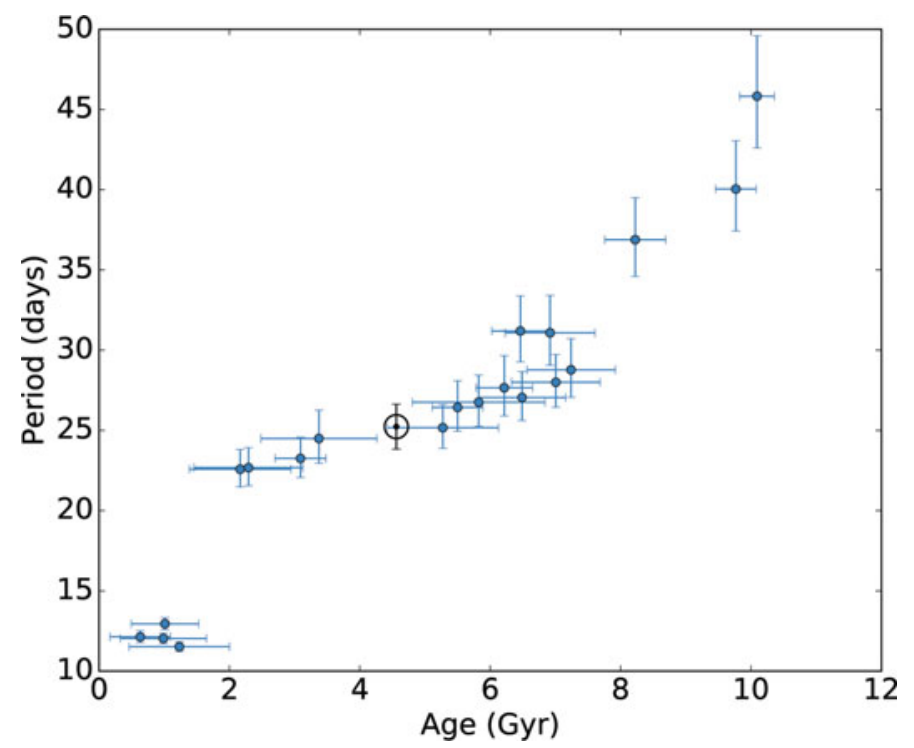

Figure 3. Evolution of inferred rotational period vs. stellar ages in solar twins, using the rotational velocities from dos Santos et al. (2016) and taking into account the variation of stellar radius with age predicted by the solar model of do Nascimento et al. (2009).

We removed all spectroscopic binaries (Fig. 1), as some of them have anomalously high $v \sin i$ for their age, likely due to interactions with their companions.

We divided the sample in bins of 2 Gyr and in each bin we only kept stars with $v \sin i$ in the upper $30 \%$, because these stars have the highest chance of having $\sin i$ above 0.9 (dos Santos et al. 2016). The selected sample is shown in Fig. 2. It is clear that the decay in rotational velocity in the first 2-3 Gyr is steeper than the Skumanich law, and that the further decline of rotational velocity with age is flatter than the Skumanich relation.

Using rotational periods from solar-type stars obtained from Kepler observations, it has been suggested that there is a weakened magnetic braking after the Sun's age (van Saders et al. 2016). We use our selected sample of solar twins with $\sin i \sim 1$, to evaluate the variation of rotational period $(P)$ with age. $P$ is obtained from our rotational velocities and the predicted variation of the solar radius with age using the model by do Nascimento et al. (2009). Our results (Fig. 3) indicate that the Sun will keep slowing down, unlike the suggestion by van Saders et al. (2016) of weakened braking for old ages. If we obtain the stellar radius using the observed $\log g$ and inferred mass, we obtain similar results but with a larger scatter. Thus, our analysis suggests that gyrochronology can be used to determine stellar ages based on observed rotational periods even for stars older than the Sun.

\section{Magnetic activity}

We study the variation of magnetic activity with age using the $\mathrm{Ca}$ II $\mathrm{H}$ and $\mathrm{K}$ lines. We measured activity indexes using triangular filters in the core of the lines and flat filters in the continuum, as in Wright et al. (2004). Our indexes were calibrated to the $S$-index of the Mount Wilson system (e.g., Baliunas et al. 1995).

We used archival data of asteroid observations from other HARPS programs to obtain the reflected solar spectra covering different epochs of the solar cycle. This guarantees that the Sun and the stars are in the same system, eliminating thus potential systematic 


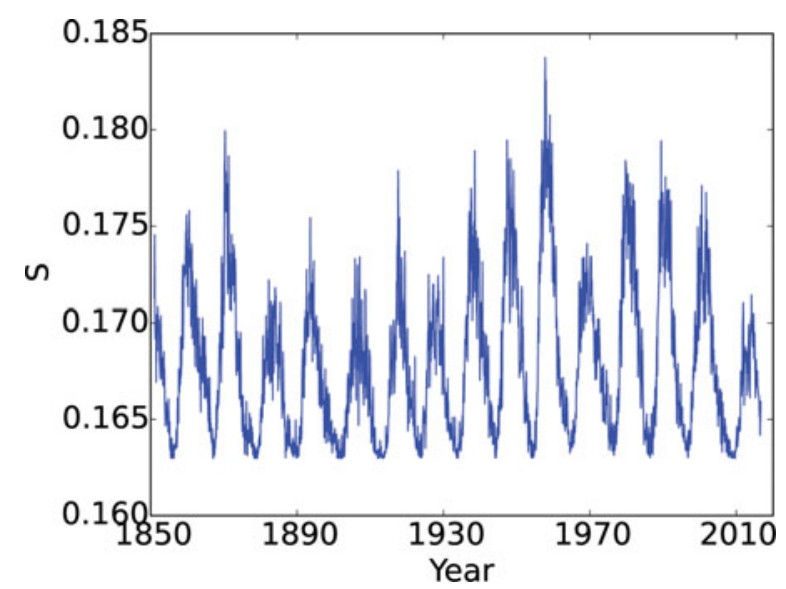

Figure 4. Evolution of the $S$-index since 1850, inferred through a calibration of the $S$-index and International Sunspot Number. Figure from Freitas et al. (2017).

problems. In order to increase the time baseline of the solar data, we calibrated the $S$-index to the International Sunspot Number, estimating the $S$-index since 1850 (Fig. 4). This allowed us to infer an accurate average solar $S$-index, considering cycles of different amplitudes.

The $S$-index is converted to the $\log \mathrm{R}_{\mathrm{HK}}$ activity index following standard procedures (e.g., Wright et al. 2004). The average of this index as a function of stellar age is shown in Fig. 5. Our data departs from the Skumanich law, suggesting a steep decrease in activity in the first 3 Gyr and then a moderate decrease of stellar activity for increasing ages.

Some spectroscopic binaries present high stellar activity for their age (Fig. 5). These stars also show high rotational velocity (Fig. 1) and chemical signatures of mass transfer, as revealed by their high yttrium abundances (Tucci Maia et al. 2016).

We obtained improved $\log \mathrm{R}_{\mathrm{HK}}$ values by using new calibrations based on $\mathrm{T}_{\text {eff }}$ rather than (B-V), as described in detail in Freitas et al. (2017). These improved values are plotted in Fig. 6. Qualitatively we obtain similar results for the decay of activity with age, but the activity-age correlation is somewhat improved, with a steep decline of activity in the first 3 Gyrs, and a shallower decrease for increasing ages. For comparison we show the relations by Donahue (1993) and Mamajek \& Hillenbrand (2008), which do not reproduce well the pattern observed in solar twins.

\section{Conclusions}

Our consistent analysis of solar twins and the Sun suggests that the evolution of rotational velocity and magnetic activity in the Sun depart from the widely used Skumanich law. The decay is steeper in the first $3 \mathrm{Gyr}$, with a slight decrease for older ages.

Our inferred longer rotation periods for increasing ages suggest that gyrochronology (e.g. Barnes 2007) is valid even beyond solar age. This result obtained with solar twins is in contrast to recent results based on solar-type stars from Kepler observations (van Saders et al. 2016). This shows the importance of having a proper comparison sample of solar twins, rather than solar-type stars that do not necessarily have the same properties as the Sun.

Furthermore, we show that the Sun is normal in rotational velocity and magnetic activity when compared to solar twins of the same age, validating thus the use of the Sun as a cornerstone calibrator in Astrophysics. 


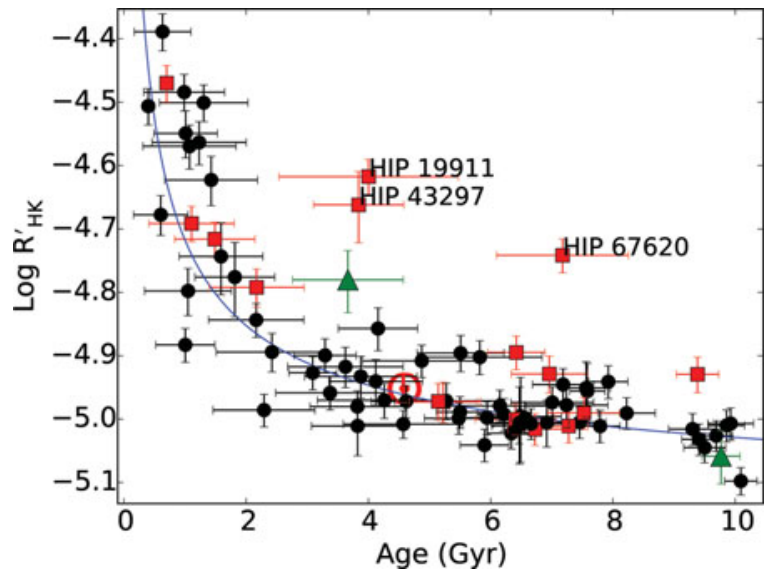

Figure 5. Log R' Hк activity index vs. age in solar twins (black circles). Spectroscopic binaries are shown by red squares (outliers are labeled). The Sun is shown with its usual symbol. Our best fit is shown. Figure from Freitas et al. (2017).

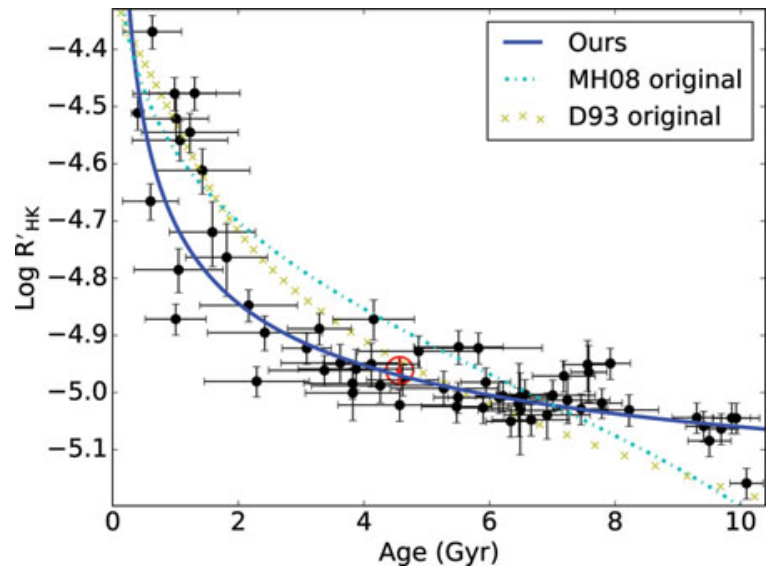

Figure 6. Log R' ${ }_{\text {H }}$ activity index vs. age in solar twins (black circles) using our improved calibrations based on $\mathrm{T}_{\text {eff }}$ (see Freitas et al. 2017). Spectroscopic binaries are excluded. Our fit is shown by the solid blue line. The other lines correspond to the relations by Mamajek \& Hillenbrand (2008) and Donahue (1993). Figure adapted from Freitas et al. (2017).

\section{References}

Adibekyan, V., Delgado-Mena, E., Figueira, P., et al. 2016, A\&3A, 591, A34

Andrássy, R. \& Spruit, H. C. 2015, A\& A, 579, A122

Baliunas, S. L., et al. 1995, ApJ, 438, 269

Baraffe, I. \& Chabrier, G. 2010, A\& $A, 521$, A44

Barnes, S. A. 2007, ApJ, 669, 1167

Baumann, P., Ramírez, I., Meléndez, J., Asplund, M., \& Lind, K. 2010, A\&̛A, 519, A87

Bazot, M., et al. 2011, A\&A, 526, L4

Bedell, M., Meléndez, J., Bean, J. L., et al. 2014, ApJ, 795, 23

Bedell, M., Meléndez, J., Bean, J. L., et al. 2015, A\& A, 581, A34

Bedell, M., Bean, J. L., Melendez, J., et al. 2016, arXiv:1611.06239

Biazzo, K., Gratton, R., Desidera, S., et al. 2015, A\& 4 , 583, A135

Carlos, M., Nissen, P. E., \& Meléndez, J. 2016, A\&A, 587, A100

Casagrande, L., Ramírez, I., Meléndez, J., Bessell, M., \& Asplund, M. 2010, A\&A, 512, A54

Casagrande, L., Portinari, L., Glass, I. S., et al. 2014, MNRAS, 439, 2060 
Castro, M., Do Nascimento, J. D., Jr., Biazzo, K., Meléndez, J., \& de Medeiros, J. R. 2011, $A \mathscr{E A}, 526, \mathrm{~A} 17$

Chambers, J. E. 2010, ApJ, 724, 92

Datson, J., Flynn, C., \& Portinari, L. 2012, MNRAS, 426, 484

Datson, J., Flynn, C., \& Portinari, L. 2014, MNRAS, 439, 1028

Datson, J., Flynn, C., \& Portinari, L. 2015, A\&SA, 574, A124

Denissenkov, P. A. 2010, ApJ, 719, 28

Desidera, S., D’Orazi, V., \& Lugaro, M. 2016, AछA, 587, A46

Donahue, R. A. 1993, Ph.D. Thesis,

Do Nascimento, J. D., Jr., Castro, M., Meléndez, J., Bazot, M., Théado, S., Porto de Mello, G. F., \& de Medeiros, J. R. 2009, A\& A, 501, 687

do Nascimento, J.-D., Jr., Takeda, Y., Meléndez, J., et al. 2013, ApJ, 771, L31

dos Santos, L. A., Meléndez, J., do Nascimento, J.-D., Jr., et al. 2016, AEGA, 592, A156

Egeland, R., Soon, W., Baliunas, S., et al. 2017, ApJ, 835, 25

Freitas, F. C., Meléndez, J., Bedell, M., et al. 2017, submitted

Gustafsson, B. 1998, Space Sci. Rev., 85, 419

Gustafsson, B. 2008, Physica Scripta, Volume T 130, pp. 014036

Jofré, P., Das, P., Bertranpetit, J., \& Foley, R. 2017, MNRAS, 467, 1140

Kawaler, S. D. 1988, ApJ, 333, 236

King, J. R., Boesgaard, A. M., \& Schuler, S. C. 2005, AJ, 130, 2318

Kiselman, D., Pereira, T. M. D., Gustafsson, B., et al. 2011, A\&3A, 535, A14

Li, T. D., Bi, S. L., Liu, K., Tian, Z. J., \& Shuai, G. Z. 2012, A\& A, 546, A83

Mahdi, D., Soubiran, C., Blanco-Cuaresma, S., \& Chemin, L. 2016, A\&SA, 587, A131

Mamajek, E. E. \& Hillenbrand, L. A. 2008, ApJ, 687, 1264

Meléndez, J., Dodds-Eden, K., \& Robles, J. A. 2006, ApJ, 641, L133

Meléndez, J. \& Ramírez, I. 2007, ApJ, 669, L89

Meléndez, J., Asplund, M., Gustafsson, B., \& Yong, D. 2009, ApJ, 704, L66

Meléndez, J., Schuster, W. J., Silva, J. S., Ramírez, I., Casagrande, L., \& Coelho, P. 2010, A\&JA, 522, A98

Meléndez, J., Ramírez, I., Karakas, A. I., et al. 2014a, ApJ, 791, 14

Meléndez, J., Schirbel, L., Monroe, T. R., et al. 2014b, A\& A, 567, L3

Meléndez, J., Bean, J. L., Bedell, M., et al. 2015, The Messenger, 161, 28

Meléndez, J., Bedell, M., Bean, J. L., et al. 2017, A\& A, 597, A34

Metcalfe, T. S., Chaplin, W. J., Appourchaux, T., et al. 2012, ApJ, 748, L10

Monroe, T. R., Meléndez, J., Ramírez, I., et al. 2013, ApJ, 774, L32

Nissen, P. E. 2015, $A \& A, 579$, A52

Nissen, P. E. 2016, $A \mathscr{E} A$, 593, A65

Pace, G. \& Pasquini, L. 2004, A\& A, 426, 1021

Pasquini, L., Biazzo, K., Bonifacio, P., Randich, S., \& Bedin, L. R. 2008, A\&AA, 489, 677

Porto de Mello, G. F. \& da Silva, L. 1997, ApJ, 482, L89

Porto de Mello, G. F., da Silva, R., da Silva, L., \& de Nader, R. V. 2014, A\&A, 563, A52

Ramírez, I., Meléndez, J., \& Asplund, M. 2009, A\&3A, 508, L17

Ramírez, I., Asplund, M., Baumann, P., Meléndez, J., \& Bensby, T. 2010, A\&AA, 521, A33

Ramírez, I., Michel, R., Sefako, R., et al. 2012, ApJ, 752, 5

Ramírez, I., Meléndez, J., Bean, J., et al. 2014, Aछ $A$, 572, A48

Ramírez, I., Khanal, S., Aleo, P., et al. 2015, ApJ, 808, 13

Robles, J. A., Lineweaver, C. H., Grether, D., et al. 2008, ApJ, 684, 691-706

Saffe, C., Flores, M., Jaque Arancibia, M., Buccino, A., \& Jofré, E. 2016, A\&\&A, 588, A81

Schirbel, L., Meléndez, J., Karakas, A. I., et al. 2015, A\& A, 584, A116

Shapiro, A. I., Schmutz, W., Cessateur, G., \& Rozanov, E. 2013, A\&A, 552, A114

Skumanich, A. 1972, ApJ, 171, 565

Soderblom, D. R. 1983, ApJS, 53, 1

Soubiran, C. \& Triaud, A. 2004, A\& A, 418, 1089

Spina, L., Meléndez, J., Karakas, A. I., et al. 2016, A\&JA, 593, A125 
Takeda, Y. \& Tajitsu, A. 2009, PASJ, 61, 471

Takeda, Y., Kawanomoto, S., Honda, S., Ando, H., \& Sakurai, T. 2007, A\&SA, 468, 663

Teske, J. K., Khanal, S., \& Ramírez, I. 2016a, ApJ, 819, 19

Teske, J. K., Shectman, S. A., Vogt, S. S., et al. 2016b, AJ, 152, 167

Tucci Maia, M., Meléndez, J., \& Ramírez, I. 2014, ApJ, 790, LL25

Tucci Maia, M., Meléndez, J., Castro, M., et al. 2015, A\&SA, 576, L10

Tucci Maia, M., Ramírez, I., Meléndez, J., et al. 2016, A\&A, 590, A32

Unterborn, C. T., Johnson, J. A., \& Panero, W. R. 2015, ApJ, 806, 139

van Saders, J. L., Ceillier, T., Metcalfe, T. S., et al. 2016, Nature, 529, 181

Wright, J. T., Marcy, G. W., Butler, R. P., \& Vogt, S. S. 2004, ApJS, 152, 261

Yana Galarza, J., Meléndez, J., Ramírez, I., et al. 2016a, A\&A, 589, A17

Yana Galarza, J., Meléndez, J., \& Cohen, J. G. 2016b, A\& A, 589, A65 